\title{
Minimum telomere length defined for healthy cells
}

How long have I got left, doctor? For a cell, at least, scientists may be close to an answer. A cell's lifespan depends on the length of its telomeres - the regions of repeating DNA that protect the ends of chromosomes. Every time a cell divides, its telomeres get shorter until they become unstable and cause chromosomes to fuse together. These fusions can make the chromosomes break when cells divide, leading to cell death or triggering genomic rearrangements associated with the early stages of cancer.

Now, Duncan Baird of the University of Cardiff, UK, and his colleagues have found the "It's important to know just how short that telomere needs to be."

The new results support the argument that it is the shortest rather than the average telomere length that counts, says Dominique Broccoli, a molecular oncologist at Memorial Health University Medical Center in Savannah, Georgia. "It's important to know just how short that telomere needs to be," she says. "It's something that people have talked about for a long time."

Although scientists suspected that this fusion might occur in otherwise healthy cells, this is the first time it has been demonstrated, says Laure Sabatier of the department of radiology and oncology at the French Atomic Energy Comshortest length telomeres can reach before they cause fusion. In addition to the steady loss associated with ageing, random mutations can cause large stochastic deletions that dramatically shrink telomeres in young, healthy cells. Baird's team focused on such cells from fetal human lung cultures whose telomeres had shrunk to the threshold length of less than 77 base pairs long. "These were normal human cells that had long, functional telomeres," says Baird. "You wouldn't expect any telomeric problems."

The critical telomere length was 12.8 repeats (of six base pairs) long, they found - any shorter and the chromosomes began to fuse together at their ends (R. Capper et al. Genes Dev. doi:10.1101/gad.439107; 2007). Baird suggests that without sufficiently long telomere caps, the cell may perceive the chromosome ends as broken strands of DNA and attempt to piece them together. mission in Fontenay-aux-Roses. "This suggests that loss of large parts of telomeres would occur even during normal cell proliferation," she says. Baird estimates that telomere fusion occurs in nearly four out of every million cells in young, healthy human cells in the lab.

The researchers also gained fresh insight into the process by which chromosomes fuse across their depleted telomeres. Each end chemically stitches together in an error-prone mechanism that can itself yield large DNA deletions. The stitching mechanism - called microhomology-mediated end joining - aligns and joins the ends of the two chromosomes via tiny sections of their complementary base-pair sequences - sometimes only six nucleotides long. This represents an interesting new pathway of telomere-driven genome rearrangements, says Broccoli.

Heidi Ledford

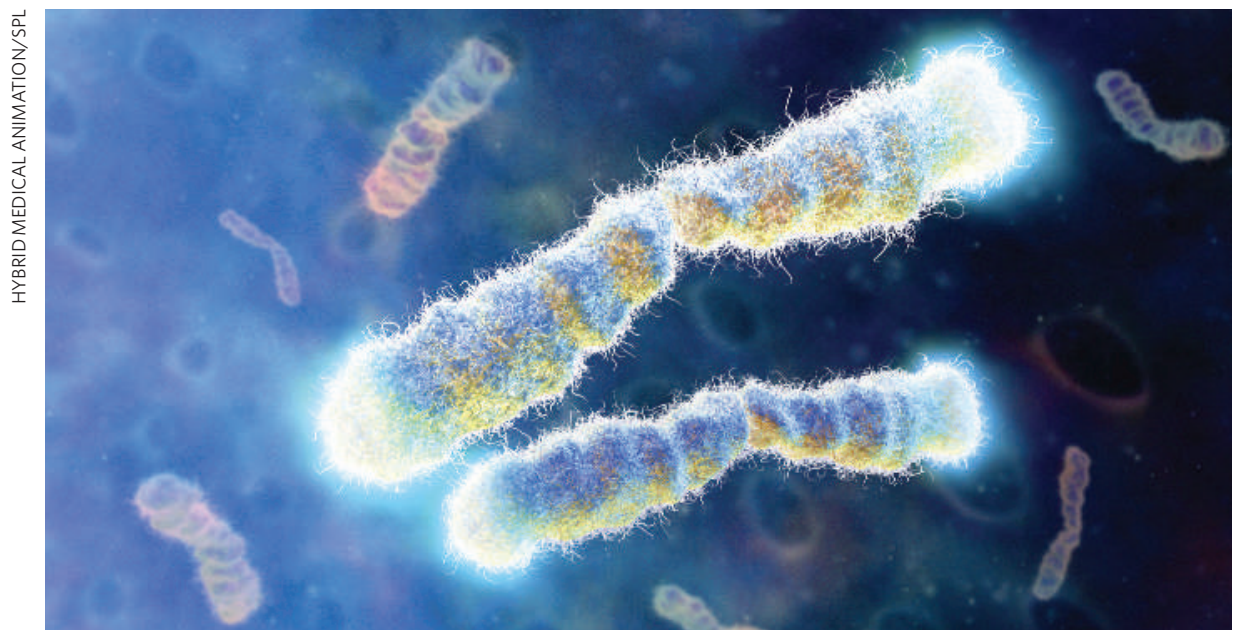

Size is important: telomeres (highlighted) protect the ends of chromosomes. 